\title{
A Rapid Lung De-cellularization Protocol Supports Embryonic Stem Cell Differentiation In Vitro and Following Implantation
}

\author{
Todd Jensen, M.Sc., ${ }^{1}$ Blair Roszell, Ph.D., ${ }^{2}$ Fan Zang, M.Sc., ${ }^{1}$ Eric Girard, M.D., ${ }^{1}$ \\ Adam Matson, M.D., ${ }^{3}$ Roger Thrall, Ph.D., ${ }^{4}$ Diane M. Jaworski, Ph.D., ${ }^{5}$ Cayla Hatton, \\ Daniel J. Weiss, M.D., Ph.D., ${ }^{6}$ and Christine Finck, M.D. ${ }^{1,2,7}$
}

Pulmonary diseases represent a large portion of neonatal and adult morbidity and mortality. Many of these have no cure, and new therapeutic approaches are desperately needed. De-cellularization of whole organs, which removes cellular elements but leaves intact important extracellular matrix (ECM) proteins and three-dimensional architecture, has recently been investigated for ex vivo generation of lung tissues. As specific cell culture surfaces, including ECM composition, profoundly affect cell differentiation, this approach offers a potential means of using de-cellularized lungs to direct differentiation of embryonic and other types of stem/progenitor cells into lung phenotypes. Several different methods of whole-lung de-cellularization have been reported, but the optimal method that will best support re-cellularization and generation of lung tissues from embryonic stem cells (ESCs) has not been determined. We present a 24-h approach for de-cellularizing mouse lungs utilizing a detergentbased (Triton-X100 and sodium deoxycholate) approach with maintenance of three-dimensional lung architecture and ECM protein composition. Predifferentiated murine ESCs (mESCs), with phenotypic characteristics of type II alveolar epithelial cells, were seeded into the de-cellularized lung scaffolds. Additionally, we evaluated the effect of coating the de-cellularized scaffold with either collagen or Matrigel to determine if this would enhance cell adhesion and affect mechanics of the scaffold. Finally, we subcutaneously implanted scaffolds in vivo after seeding them with mESCs that are predifferentiated to express pro-surfactant protein C (pro-SPC). The in vivo environment supported maintenance of the pro-SPC-expressing phenotype and further resulted in vascularization of the implant. We conclude that a rapid detergent-based de-cellularization approach results in a scaffold that can maintain phenotypic evidence of alveolar epithelial differentiation of ESCs and support neovascularization after in vivo implantation.

\section{Introduction}

$\mathbf{M}$ ANY DEVASTATING ADULT and pediatric pulmonary diseases remain without cure and without effective treatments. Additionally, in 2008, one in eight babies $(12.3 \%$ of live births) was born preterm in the United States. ${ }^{1,2}$ These births are complicated by premature lungs and may be associated with pulmonary hypoplasia. ${ }^{1}$ There is no effective treatment, but the ability to utilize stem cells postnatally to generate functional alveolar tissue may help ameliorate this disease process. We previously published a two-step procedure for differentiating murine embryonic stem cells
(mESCs) into cells with phenotypic characteristics of type II alveolar epithelial cells using standard tissue culture techniques. ${ }^{3}$ Other laboratories have also generated alveolar epithelial type II cells (AEII) cells from mESCs, but, in general, the yield is low. ${ }^{3-8}$ The ability to improve yield of functional AEII cells from mESCs by using alternative culture systems, such as three-dimensional (3D) biomimetic scaffolds, would enhance the potential clinical applicability for pulmonary diseases.

A growing body of evidence demonstrates that specific cell culture surfaces, including composition of extracellular matrix (ECM) proteins, stiffness, and elasticity, profoundly

Departments of ${ }^{1}$ Vascular Biology and ${ }^{2}$ Cell Biology, University of Connecticut Health Center, Farmington, Connecticut.

${ }^{3}$ Department of Neonatology, Connecticut Children's Medical Center, Hartford, Connecticut.

${ }^{4}$ Department of Immunology, University of Connecticut Health Center, Farmington, Connecticut.

Departments of ${ }^{5}$ Anatomy \& Neurobiology and ${ }^{6}$ Pulmonary and Critical Care Medicine, University of Vermont College of Medicine, Burlington, Vermont.

${ }^{7}$ Department of Surgery, Connecticut Children's Medical Center, Hartford, Connecticut. 
Table 1. Reducing the 50-h De-Cellularization Protocol to 24-h While Still Using the SAme Reagents

\begin{tabular}{lcccc}
\hline & $\begin{array}{c}0.1 \% \\
\text { Triton-X100 }\end{array}$ & $\begin{array}{c}2 \% \\
\text { SDC }\end{array}$ & $\begin{array}{c}1 \mathrm{M} \\
\mathrm{NaCl}\end{array}$ & $\begin{array}{c}30 \mu \mathrm{g} / \mathrm{mL} \\
\text { DNAse }\end{array}$ \\
\hline 50-h protocol & $24 \mathrm{~h}$ & $24 \mathrm{~h}$ & $1 \mathrm{~h}$ & $1 \mathrm{~h}$ \\
24-h protocol & $8 \mathrm{~h}$ & $14 \mathrm{~h}$ & $1 \mathrm{~h}$ & $1 \mathrm{~h}$ \\
\hline
\end{tabular}

Duration of each detergent used in the 50-h and 24-h decellularization process is detailed above. The 24-h protocol was still effective in de-cellularizing the normal lung despite the time reduction in the first two steps.

SDC, sodium deoxycholate.

affect mESC differentiation. ${ }^{9-13}$ Furthermore, a number of recent studies have attempted to mimic the unique lungspecific 3D matrix using a variety of biomimetic matrices and degradable scaffolds such as collagen and gelfoam. ${ }^{10,14,15}$ An inherent problem with these systems is the inability to recapitulate the complex 3D architecture of the lung. Most recently, an old technique for de-cellularizing intact whole lungs to remove all cellular materials leaving a 3D matrix has been re-investigated. ${ }^{14,16,17}$ This 50 -h de-cellularization procedure utilizes Triton-X100 and sodium deoxycholate (SDC) to remove cells and leaves behind a 3D ECM. Additionally, two recent studies utilizing 2-h de-cellularization approaches with different detergents (SDS or CHAPS) supported cellular growth; however, the resulting cellular architecture did not closely resemble that of the native lung. ${ }^{18-20}$ Therefore, the optimal de-cellularization protocol that maintains 3D architecture has not been defined.

An important characteristic of scaffolds is that they support cellular differentiation. One report suggests that inoculation of de-cellularized rat lungs with undifferentiated mESCs could result in a variety of mature lung cell types. ${ }^{10}$ This decellularization protocol required several weeks and utilized both physical (multiple freeze-thaws) as well as a detergentbased (SDS) de-cellularization. We present a simplified 24-h approach to de-cellularization compared to recently published 50-h protocols utilizing Triton/SDC. ${ }^{14,17}$ This shortened protocol demonstrates maintenance of 3D lung architecture and ECM protein compostion. After inoculating with predifferentiated mESCs, these de-cellularized scaffolds maintained expression of pro-surfactant protein C (pro-SPC) and thyroid transcription factor 1 (TTF1). Manipulating the scaffolds by coating with collagen and Matrigel did not appear to affect 3D architecure, cellular adhesion, or phenotypic expression of mESCs. When implanted subcutaneously, the constructs maintained phenotypic expression of distal alveolar cells while supporting neovascularization from the host.

\section{Methods}

\section{De-cellularization process (Table 1)}

Adult male and female C57/BL6 mice were used as lung donors for the de-cellularization process. Mice were euthanized in accordance with UCHC IACUC-approved protocols. A midline incision was made along the throat, and the trachea was exposed. A small incision was made in the trachea, and an 18-gauge tubing adapter (Becton Dickinson) was inserted and secured. Once excised from the mouse, the lungs were rinsed in deionized $\mathrm{H}_{2} \mathrm{O} / 5 \times$ PenStrep (rinse solution).
Twenty-four-hour de-cellularization protocol. After removal of the intact heart-lung bloc, $0.1 \%$ Triton-X100 was instilled into the trachea, and the lungs were incubated for $8 \mathrm{~h}$ at room temperature. After $8 \mathrm{~h}$, the lungs were rinsed and treated with $2 \%$ SDC for $14 \mathrm{~h}$ at $4^{\circ} \mathrm{C}$. The lungs were rinsed and treated with $1 \mathrm{M}$ sodium chloride for $1 \mathrm{~h}$ at room temperature. Lastly, lungs were rinsed and treated with a solution containing $30 \mu \mathrm{g} / \mathrm{mL}$ porcine pancreatic DNAse for $1 \mathrm{~h}$ at room temperature. Lungs were flushed with phosphate-buffered saline (PBS) before they were utilized for experiments.

Fifty-hour protocol. Lungs were similarly treated with $0.1 \%$ Triton-X100 for $24 \mathrm{~h}$ at $4^{\circ} \mathrm{C}$. After $24 \mathrm{~h}$, the lungs were rinsed and treated with $2 \%$ SDC for $24 \mathrm{~h}$ at $4^{\circ} \mathrm{C}$. The lungs were rinsed and treated with $1 \mathrm{M}$ sodium chloride for $1 \mathrm{~h}$ at room temperature. Lastly, lungs were rinsed and treated with a solution containing $30 \mu \mathrm{g} / \mathrm{mL}$ porcine pancreatic DNAse for $1 \mathrm{~h}$ at room temperature. Lungs had been flushed with PBS before they were utilized for experiments.

\section{Histology, alveolar morphogenesis, and nuclei count}

De-cellularized whole lungs were gravity-fixed for $1 \mathrm{~h}$ in $4 \%$ paraformaldehyde. Hematoxylin \& eosin (H\&E) staining was used to visually quantify the nuclei loss by comparing nuclei counts in 5- $\mu \mathrm{m}$ paraffin sections of normal and decellularized lungs. Nuclei were counted from three random images at $400 \times$ magnification using a counting tool (Adobe Photoshop CS5; Adobe Systems). Data were statistically analyzed using GraphPad Prism Software via one-tail $t$-test with $p \leq 0.05$. Alveolar structures were quantified by comparing the mean cord length (MCL) between conditions using established, previously published morphometric techniques. ${ }^{21}$ At least, five random fields of each condition were imaged with $400 \times$ magnification on a Zeiss microscope. Images containing nonalveolar structures such as bronchi were not used. For the MCL, at least five lungs were analyzed for each condition. Each image was overlaid with a grid generated using ImageJ $(\mathrm{NIH}){ }^{21}$ The average length of each line through airspaces was summed and averaged as the MCL.

\section{Electron microscopy}

Normal and 24-h de-cellularized lungs were gravity-fixed immediately following de-cellularization protocols in $2.5 \%$ glutaraldehyde in $0.1 \mathrm{M}$ cacodylate buffer. Samples were infused with the fixative and then immersed in the solution at $4^{\circ} \mathrm{C}$ for 1 day before analysis. High-power and low-power images were obtained to compare the ECM composition and ECM appearance before and after the 24-h de-cellularization protocol.

\section{Western blots}

Western blot analysis using $25 \mu \mathrm{g}$ protein (whole-cell lysate) with antibodies against actin, collagen type I, collagen type IV, fibronectin, glyceraldehyde 3-phosphate dehydrogenase (GAPDH), histone H1, laminin, and smooth muscle myosin was performed as previously described. ${ }^{17,22,23}$ Protein expression following 24-h de-cellularization protocols (Fast, Triton alone, SDC alone) and the 50-h de-cellularization protocol (TritonSDC- and PBS-rinsed lungs as a control) was evaluated on two separate blots; thus, expression was normalized to the naïve lung on each respective blot. Densitometry was performed using 


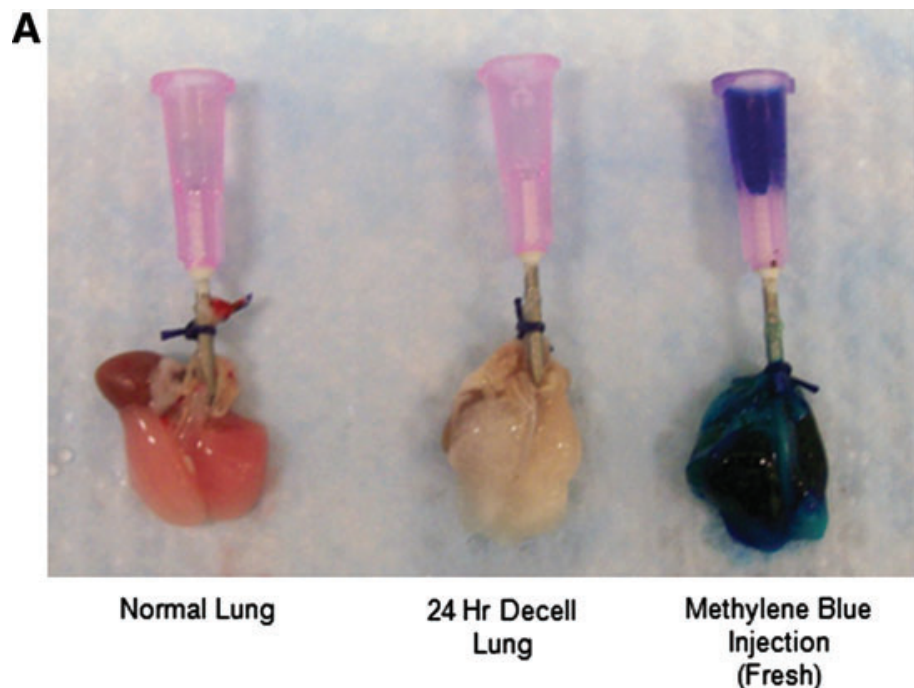

B
24 Hour Decell
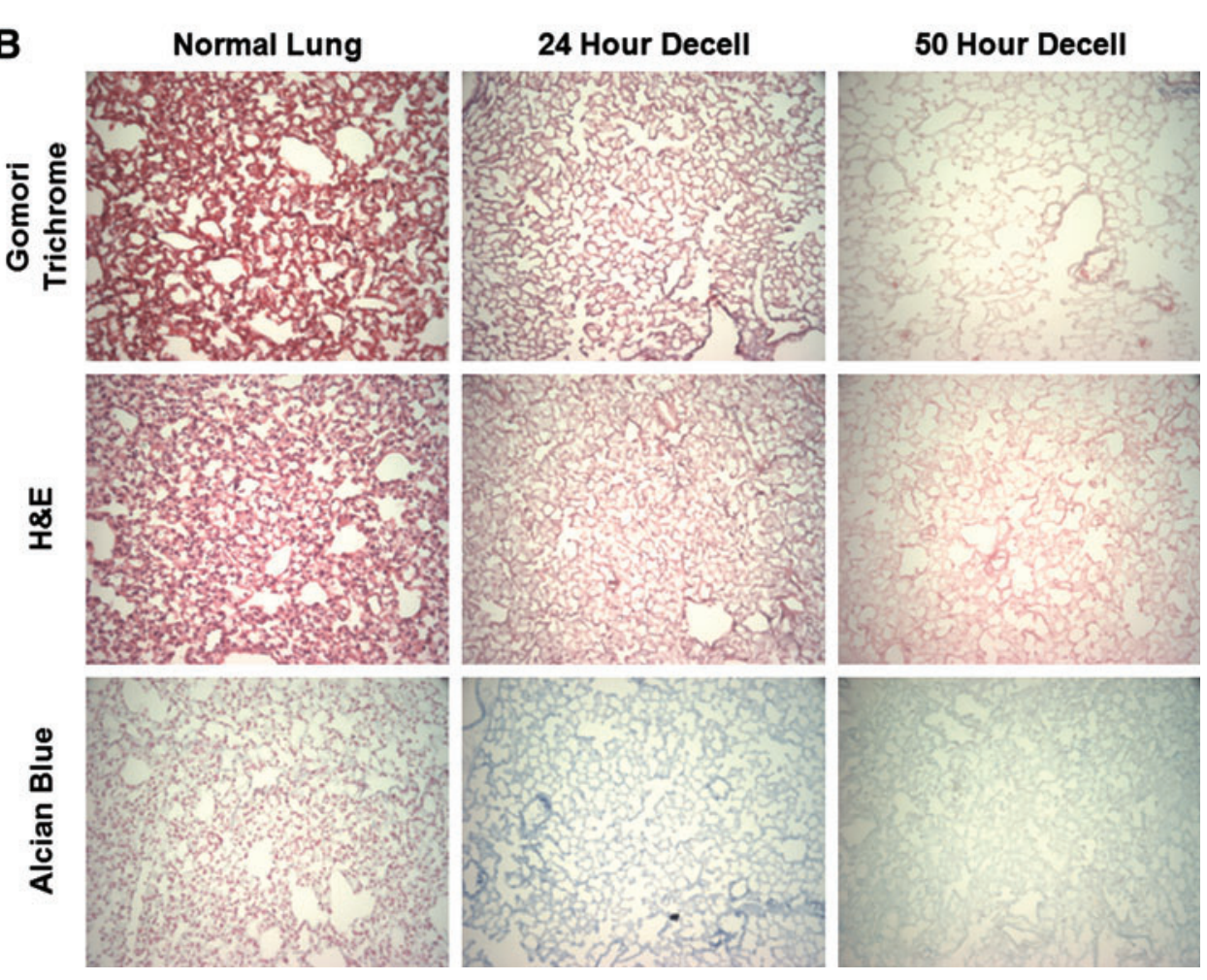

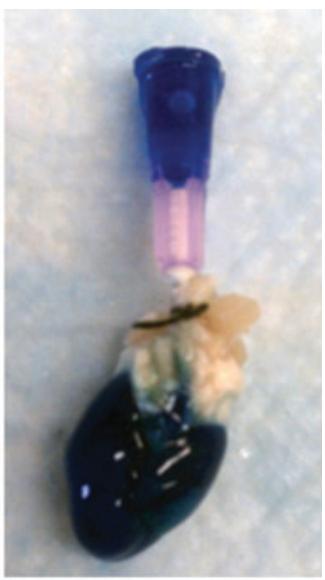

Methylene Blue Injection (7 Days PostDecell)

FIG. 1. Twenty-four-hour de-cellularization protocol effectively removes cells and maintains architecture but increases alveolar spacing. (A) Gross histology of the normal and de-cellularized lung. The addition of methylene blue demonstrates patency of the construct immediately and 7 days after de-cellularization. (B) Histology of 50-h and 24-h de-cellularization protocols compared to the normal lung with gomori trichrome, H\&E, and alcian blue staining. Representative images for all conditions are shown ( $20 \times$ magnification). (C) Nuclei count and the alveolar mean cord length are calculated after 24 -h de-cellularization $\left(n=5 .{ }^{*} p<0.05\right)$. (D) Transmission and scanning electron microscopy of the 24-h de-cellularized lung compared to the normal lung. H\&E, hematoxylin \& eosin. Arrows indicating disrupted basement membrane. Color images available online at www.liebertonline.com/tec

Quantity One software (Bio-Rad). Differences in detergent and/ or timing of detergent treatment were assessed by one- or twoway analysis of variance using Prism software (Graph Pad).

\section{Gelatinase assay}

The net gelatinolytic activity was determined using the EnzCheck gelatinase assay (Molecular Probes) as previously described. ${ }^{23}$ Lungs were homogenized in tris-buffered saline (50 mM Tris- $\mathrm{HCl}, \mathrm{pH} 7.4 ; 150 \mathrm{mM} \mathrm{NaCl}$ ), and the gelatinolytic activity (in $80 \mu \mathrm{g}$ protein) was reported as the rate of fluorescence increase over $4 \mathrm{~h}$ normalized to an assay reagent containing DQ gelatin alone. The specificity of the assay was determined by the inclusion of the matrix metalloproteinase (MMP) inhibitor 1,10 phenanthroline $(1 \mathrm{mM})$ or a cocktail of protease inhibitors (phenyl-methyl sulfonylfluoride [1 $\mathrm{mM}]$ 

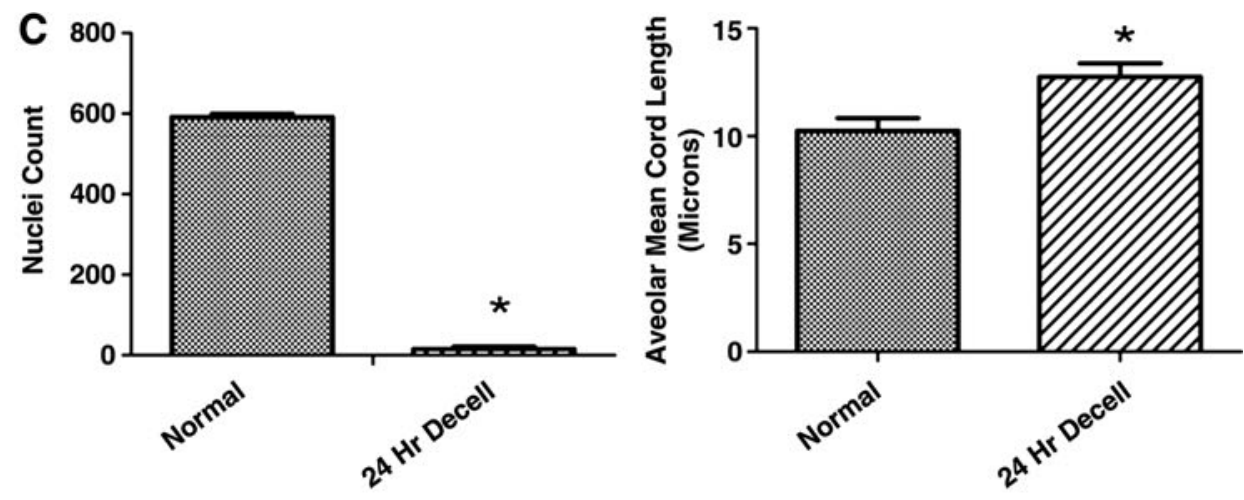

D
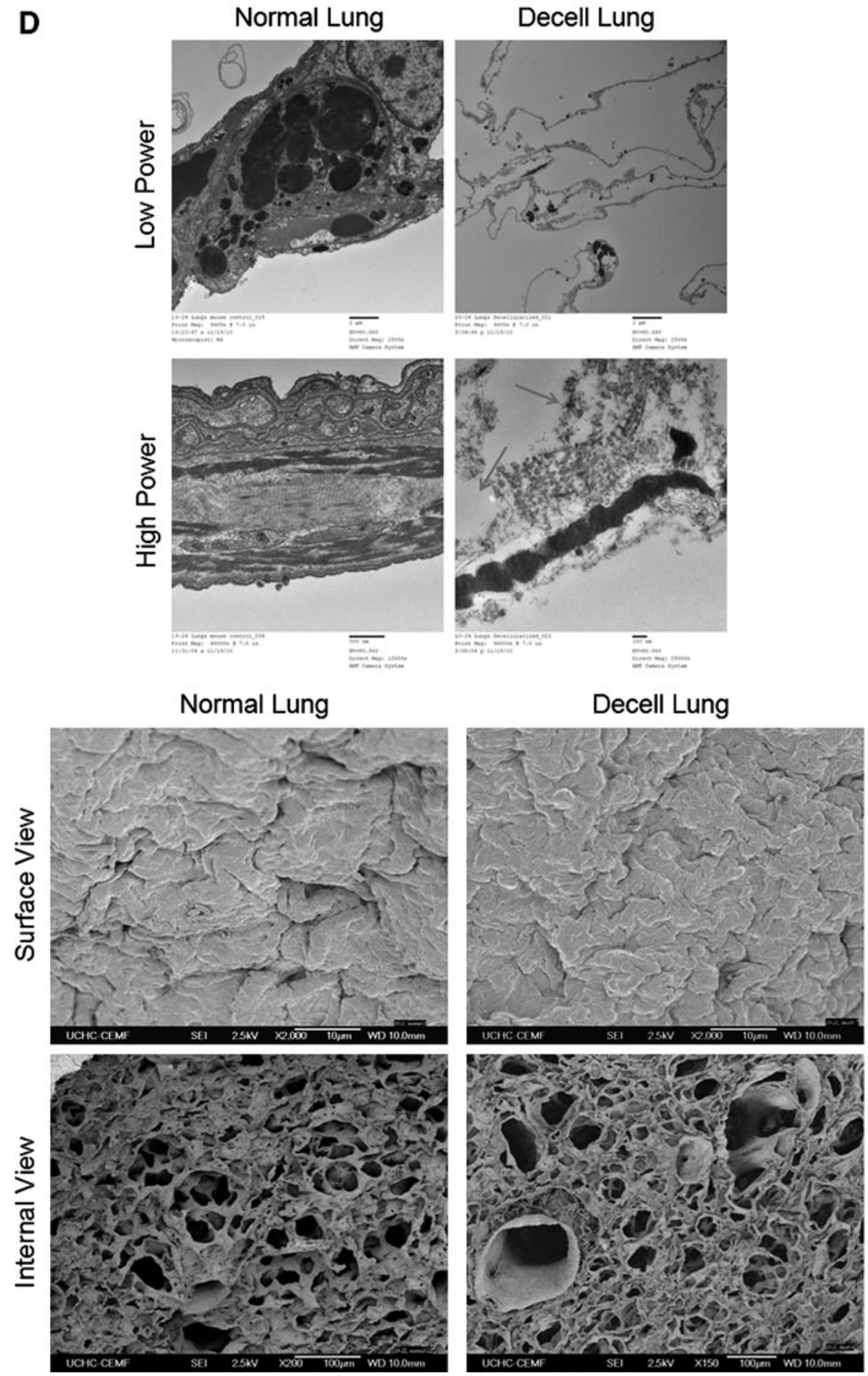

FIG. 1. (Continued). 
FIG. 2. Comparison of the protein content and protease activity between the $50-\mathrm{h}$ and 24-h de-cellularization protocols shows an increased protease activity in the 24-h protocol. (A) Western blot analysis ( $25 \mu \mathrm{g}$ whole-cell lysate) of lungs de-cellularized with the 24-h (fast) or 50-h (Triton-SDC [TSDC]) protocol (right lane in each blot) relative to freshly dissected naïve lungs (left lane in each blot). (B) Net proteolytic activity in lungs de-cellularized for $24 \mathrm{~h}$ (complete fast protocol compared to Triton X-100 or SDC alone) or $50 \mathrm{~h}$ (complete Triton-SDC protocol compared to control PBS-rinsed lungs) relative to freshly dissected naïve lungs $(C=$ lungs treated with PBS, T = lungs treated with the 24-h de-cellularization protocol). Data are presented as mean \pm standard error of the mean $(n=3)\left({ }^{*} p<0.05\right.$, $* * \leq 0.01, \# p \leq 0.001$ \#\# 1 0.0001). GAPDH, glyceraldehyde 3-phosphate dehydrogenase; PBS, phosphate buffered saline; SDC, sodium deoxycholate.
A
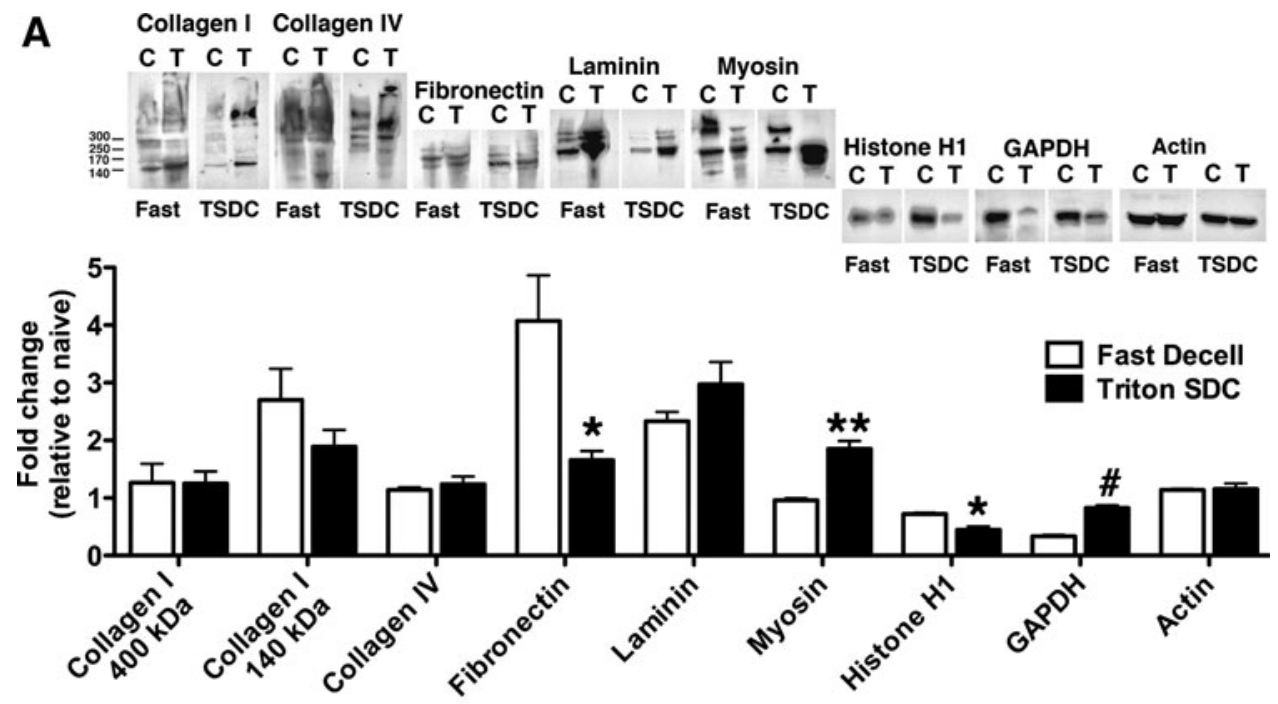

B

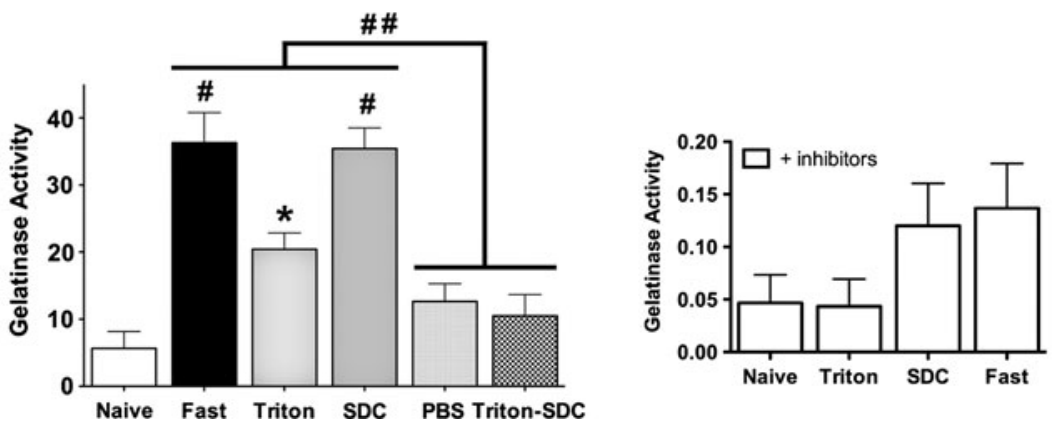

to inhibit serine protease, $\mathrm{N}$-ethylmaleimide and $\varepsilon$-amino- $n$ caproic acid [ $5 \mathrm{mM}$ each] to inhibit cysteine proteases, pepstatin A $[5 \mu \mathrm{g} / \mathrm{mL}]$ to inhibit acid proteases, and leupeptin $[5 \mu \mathrm{g} / \mathrm{mL}]$ to inhibit serine and cysteine proteases). ${ }^{22,23}$

\section{Immunofluorescence}

De-cellularized lung scaffolds were gravity-fixed in $4 \%$ paraformaldehyde followed by paraffin embedding. Sections were deparafinized, permeabilized, and serum-blocked in the PBS containing $0.1 \%$ Triton-x (Sigma) and 1\% bovine serum albumin (Fisher Scientific) and 1\% Tween-20 (Biorad). The primary antibody was applied overnight at $4^{\circ} \mathrm{C}$ (see Supplementary Data; Supplementary Data are available online at www.liebertonline.com/tec). As a control, the primary antibodies were omitted to assess for nonspecific binding. The secondary antibody (Donkey Anti-Rabbit Alexa 594 or Donkey Anti-Goat Alexa 488; Invitrogen) was applied at room temperature. Samples were counterstained with Topro-3 (Invitrogen) or propidium iodide (Abcam) and mounted with Shandon Immuno-Mount (Thermo-Scientific). Samples were imaged using a Zeiss Axiovert 200M Confocal Microscope with an LSM 510 Meta Laser Module and Carl Zeiss LSM Image Browser Software (Carl Zeiss MicroImaging, LLC). The same imaging software was used to process the images in addition to using Adobe Photoshop CS5 (Adobe Systems). The positive cell count was normalized to the total cell count in a given image using the counting tool in Adobe Photoshop (Adobe Systems), and Graph Pad Prism software (Graph Pad) was utilized to generate graphs for the data.

\section{Coating of de-cellularized scaffolds}

Lungs were coated through the trachea with either collagen I or Matrigel ${ }^{\mathrm{TM}}$ (BD Biosciences). Collagen I was diluted in PBS to a concentration of $0.8 \mathrm{mg} / \mathrm{mL}$ before inoculation, and Matrigel was also diluted in PBS to a concentration of $0.4 \mathrm{mg} / \mathrm{mL}$. A coating agent was injected through the trachea into all lobes of the scaffold until they were visually fully expanded followed by incubation for $20 \mathrm{~min}$ at $37^{\circ} \mathrm{C}$. Since collagen I and Matrigel were diluted from stock concentration, they did not solidify in the lung.

\section{Cell culture techniques}

Murine ESCs, E14tg2a (American Type Culture Collection), were maintained under feeder-free conditions in a culture medium (maintenance medium), which was comprised of the Glasgow minimum essential medium (GMEM; Invitrogen), 10\% batch-tested fetal bovine serum (Biowest), $5 \mathrm{~mL}$ sodium pyruvate $(1 \mathrm{mM}$; Gibco), $5 \mathrm{~mL}$ nonessential amino acids $(1 \mathrm{mM}$; Gibco), $5 \mathrm{~mL}$ glutamine ( $2 \mathrm{mM}$; Invitrogen), $50 \mathrm{U} / \mathrm{mL}$ penicillin, $50 \mathrm{mg} / \mathrm{mL}$ streptomycin (Invitrogen), leukemia inhibitory factor $1000 \mathrm{U} / \mathrm{mL}$ (ESGRO; Millipore), and $0.1 \mathrm{mM} \mathrm{b}$-mercaptoethanol (Sigma). For routine maintenance and propagation, the cells were split in $0.1 \%$ gelatin-coated T-25 flasks (Falcon-Beckton Dickinson) every 2-3 days using TrypLE (Gibco), re-suspended in the maintenance medium, and re-plated. E14tg2a cells were 

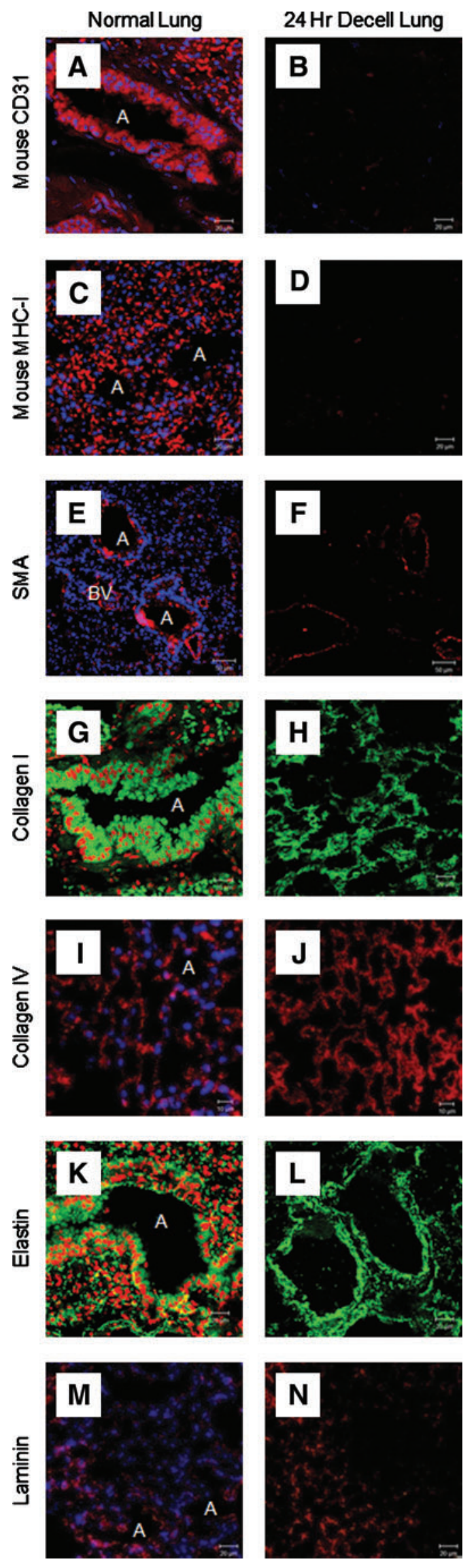

induced to differentiate using the dissociated seeding method. ${ }^{3}$ Cells were trypsinized, disaggregated into a single-cell suspension, and plated at a low density $\left(3 \times 10^{4}\right.$ cells $\left./ \mathrm{cm}^{2}\right)$. This density allowed for cell expansion for up to 8 days without passaging. For directed differentiation to endoderm, all media were serumfree and supplemented with activin A $(20 \mathrm{ng} / \mathrm{mL} ; \mathrm{R} \& \mathrm{D}$ Systems) and Wnt3a (10 ng/mL; R\&D Systems) per our previously published protocol. To promote differentiation into cells with phenotypic characteristics of AEII cells (pro-SPC expression), ${ }^{3}$ the medium was changed to a serum-free medium supplemented with $50 \mathrm{mg} / \mathrm{mL}$ heparin sulfate salt (porcine intestinal mucosal; Sigma) and 50 ng/mL of FGF2 (Cat\# F-0291; Sigma). Supplementary Figure S2 summarizes the steps of differentiation.

\section{Seeding of de-cellularized scaffold with differentiated mESCs}

Four to eight million cells per lung were suspended in media at a volume of $400-500 \mu \mathrm{L}$ and kept on ice until seeding. Cell viability was assessed using trypan blue exclusion during counting. In a separate experiment (data not shown), a cell viability assay was performed, indicating qualitatively $>90 \%$ viability (Invitrogen Live/Dead Assay). After rinsing the lungs in PBS and following the previously detailed coating procedure, the cell suspension was drawn up into a 1-cc syringe and slowly injected into the lung scaffolds through the tracheal cannula. Seeded lung scaffolds were submerged in $4-5 \mathrm{~mL}$ of differentiation media and allowed to incubate for $24 \mathrm{~h}$. Supplementary Figure S2 provides a summary of the steps in seeding the scaffolds.

\section{Mechanical properties of de-cellularized lungs}

Lung mechanics were measured using a computer-controlled small animal ventilator (FlexiVent; SCIREQ), which is typically used for pulmonary function testing in anesthetized and tracheostomized mice and more recently in de-cellualrized lung scaffolds. ${ }^{14,24}$ The FlexiVent was calibrated for open and closed tube systems for each pulmonary test performed. Cannulated lungs were connected to the FlexiVent using a frequency of 150 breaths/min at a tidal volume of $7 \mathrm{~mL} / \mathrm{kg}$. The maximum pressure was set to $20-\mathrm{cm}_{2} \mathrm{O}$ with a positive-end expiratory pressure of $3-\mathrm{cm} \mathrm{H}_{2} \mathrm{O}$. Ventilation occurred with lungs suspended in saline, and the development of air leak was identified by bubbles exiting from the lungs into the fluid phase. Lungs that developed air leak were excluded from analysis. Pressure, flow, and volume were used to calculate total lung compliance, elastance, and total lung resistance. Three normal lungs and three decellularized scaffolds were tested, and a total of 15 measurements were recorded and averaged per lung/scaffold to obtain the final values.

\footnotetext{
FIG. 3. An immunofluoresence analysis of a de-cellularized scaffold following the 24-h de-cellularization protocol shows preservation of ECM proteins with loss of endothelial cell and MHC class I expression. Mouse CD31 (A, B), mouse MHC Class I $(C, D)$, SMA (E, F), collagen I $(G, H)$, collagen IV $(I, J)$, elastin $(\mathbf{K}, \mathbf{L})$, and laminin $(\mathbf{M}, \mathbf{N})$. Representative images for all conditions are shown $(63 \times$ oil magnification). ECM, extracellular matrix; SMA, smooth muscle actin; A, airway; BV, blood vessel. Color images available online at www.liebertonline.com/tec
} 

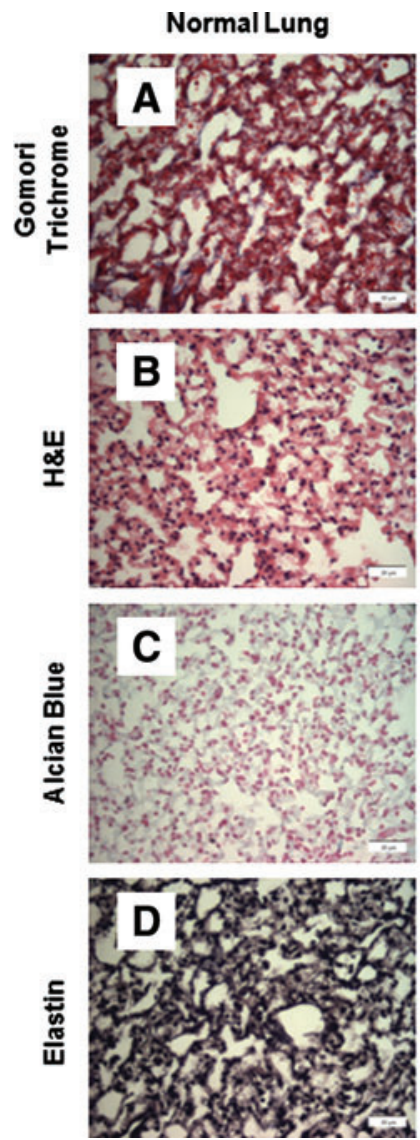

$24 \mathrm{Hr}$ Decell Lung
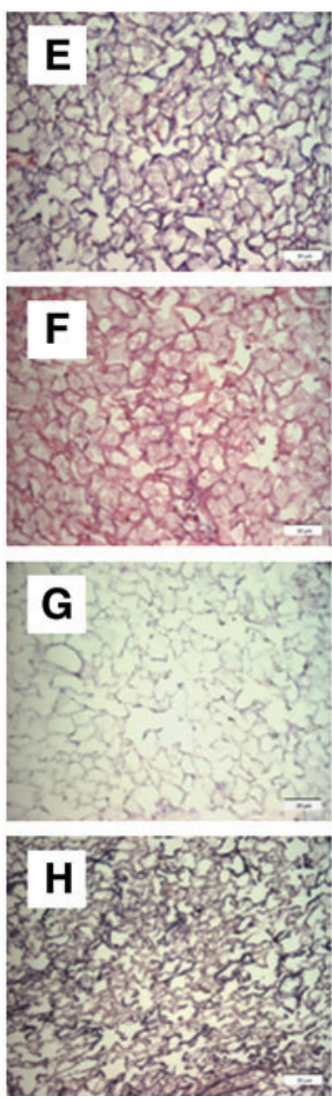

Matrigel Coated
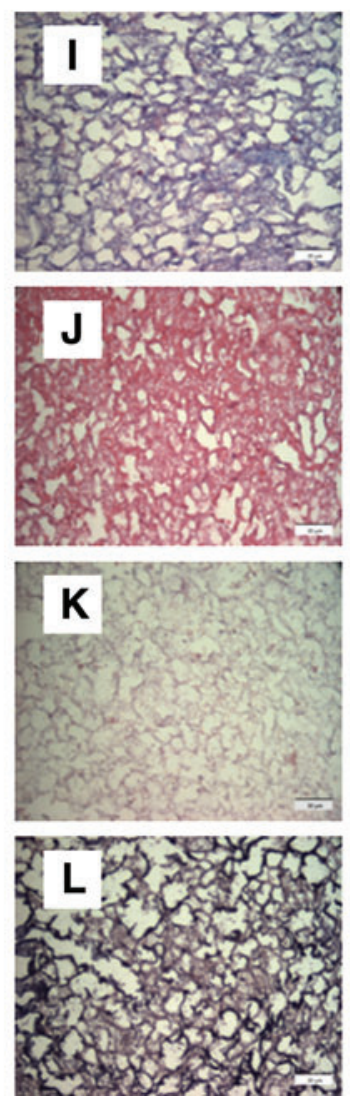

\section{Collagen Coated}
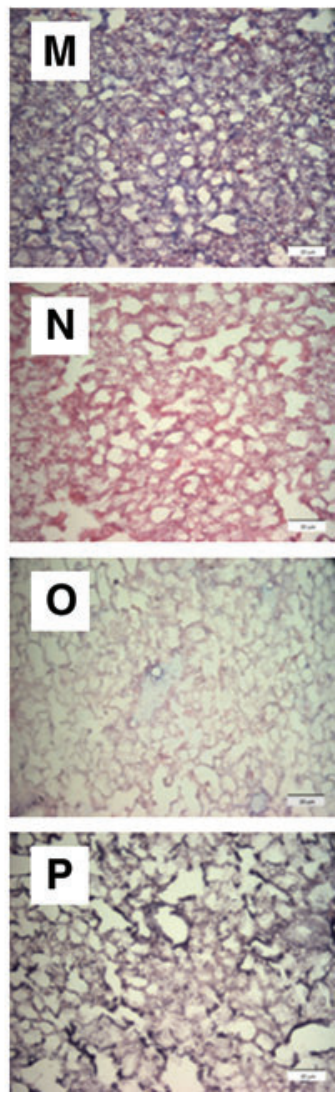

FIG. 4. Addition of coating agents to the 24-h de-cellularized lung. Gomori trichrome staining (A, E, I, M) was utilized to qualitatively assess collagen levels compared to normal lung. Hemotoxylin and eosin staining (B, F, J, N) was utilized to qualitatively assess matrix and any remaining nuclei. Alcian blue $(\mathbf{C}, \mathbf{G}, \mathbf{K}, \mathbf{O})$ assessed the amount of glycosaminoglycans present in all conditions compared to normal lung. Elastin staining (D, H, L, P) demonstrated elastin levels in all conditions compared to normal lung. Representative images for all conditions are shown $(40 \times$ magnification $)$. Color images available online at www.liebertonline.com/tec
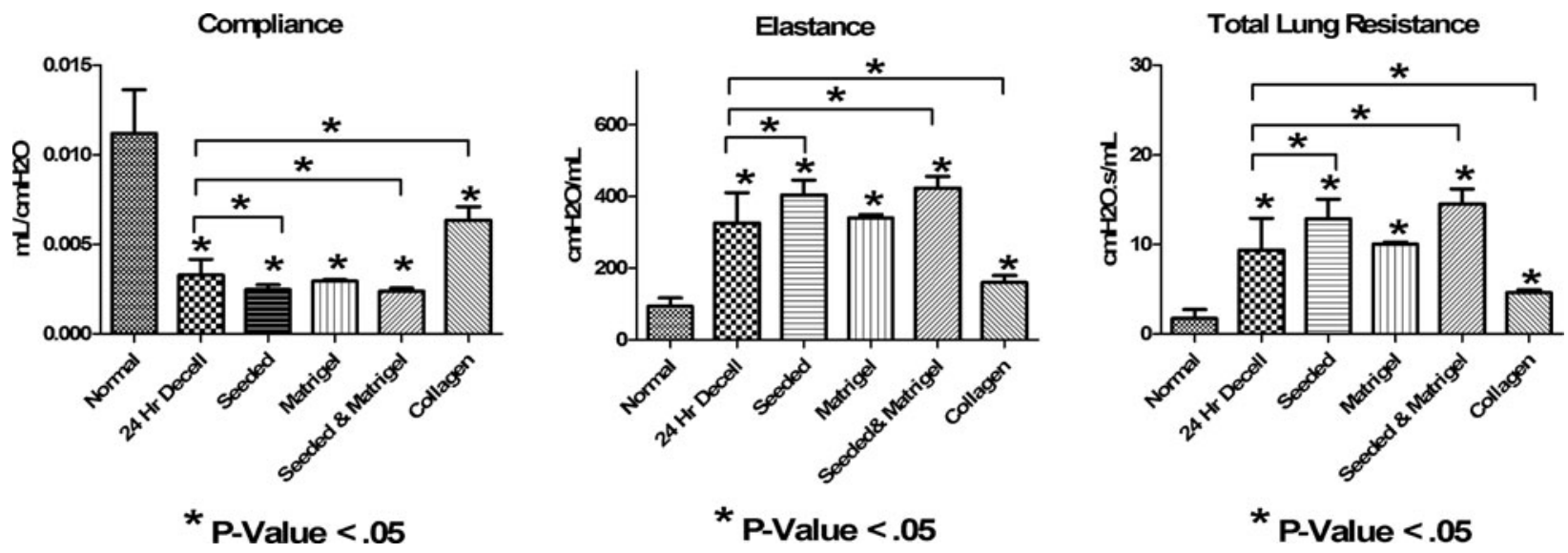

FIG. 5. Mechanical analysis of 24-h de-celluarized, seeded, and coated scaffolds shows increased resistance and elastance, and decreased compliance. Mechanics of the normal lung and 24-h de-cellularized lung (24-h decell), 24-h de-cellularized lung seeded with differentiated mESCs (seeded), 24-h de-cellularized lung coated with Matrigel ${ }^{\mathrm{TM}}$ (Matrigel), 24-h decellularized lung coated with Matrigel and seeded with mESCs (seeded and Matrigel), and 24-h de-cellularized lung coated with collagen I (collagen). Data demonstrate that the de-cellularization process results in decreased compliance, increased total lung resistance, and increased elastance consistent with loss of surfactant and other ECM components $\left(n=3\right.$; $\left.{ }^{*} p<0.05\right)$. mESCs, murine embryonic stem cells. 


\section{Implantation of lung constructs and evaluation of vessel formation}

Constructs were seeded with 4-8 million viable differentiated mESCs and allowed to incubate in culture for $24 \mathrm{~h}$. The constructs were subcutaneously implanted in SCID/beige mice for 14 days to evaluate for maintenance of differentiation and blood vessel formation within the constructs (Supplementary Fig. S3). At the time of harvest, FITC dextran (Sigma-Aldrich) was injected $(50 \mathrm{mg} / \mathrm{mL})$ via tail vein and allowed to circulate for $5 \mathrm{~min}$ before euthanizing the animal.

\section{Results \\ Characteristics of the de-cellularized lung: 50- $h$ versus $24-h$ protocol}

Table 1 summarizes the steps of the two de-cellularization processes. Grossly, the lungs become more translucent with decellularization (Fig. 1A). Additionally, instillation of methylene blue through the trachea immediately after the de-cellularization process depicted an intact scaffold without extravasation of the dye. This suggests that the de-cellularization process maintains the integrity of the ECM proteins. After 7 days of PBS storage, the scaffold still appeared intact with only minor indication of leak noted after dye instillation (Fig. 1A).

This 24-h de-cellularization process, utilizing detergents, hypertonic saline, and DNAse, preserved the architecture of the lung while eliminating the nuclei. Figure 1B and Supplementary Figure S4 depict retention of collagen in blue on gomori trichrome staining (Fig. 1B and Supplementary Fig. S4), with preserved architecture and matrix on H\&E staining (Fig. 1B and Supplementary Fig. S4). There was a considerable loss of glycosaminoglycans (GAGs), as evident by alcian blue staining (Fig. 1B and Supplementary Fig. S4) similar to that observed in other studies. ${ }^{14,19}$ GAGs are complex sugars often connected to proteins and contribute to the viscoelastic behavior of parenchymal lung tissue. ${ }^{25}$ In addition, a significant reduction in the nuclei count resulted in $<15$ nuclei present per tissue section, indicating significant de-cellularization. Also, an increase in the MCL (Fig. 1C) was evident, indicating preservation of the airway structure.

\section{Transmission and scanning electron microscopy of the de-cellularized scaffold}

Transmission electron microscopy imaging of the normal lung shows a type II alveolar cell with lamellar bodies present and an undamaged basement membrane (Fig. 1D). The 24-h de-cellularized scaffold shows a denuded membrane with no cellular debris (Fig. 1D); however, some distortion to the basement membrane is noted (Fig. 1D, red arrows). Scanning electron microscopy imaging revealed no large visual differences of the internal view after de-cellularization (Fig. 1D). Furthermore, the surface appears clear and intact.

\section{Analysis of the ECM protein content and proteolytic activity}

Western blot analysis of the ECM (collagen I, collagen IV, fibronectin, and laminin), cytosolic (smooth muscle myosin, GAPDH, and actin), and nuclear (histone H1) proteins subsequent to 24-h (fast decell) or 50-h (Triton/SDC) decellularization were compared to quantify the efficiency of the shortened protocol (Fig. 2A). For the vast majority of proteins analyzed, both de-cellularization protocols were comparable. However, the fast protocol was more effective in removal of cytosolic proteins (GAPDH and myosin) and resulted in increased fibronectin content, while the 50-h protocol was more effective in removal of the nuclear protein histone H1. These results suggest that a comparable scaffold can indeed be generated in the 24-h protocol, compared to the 50-h protocol, while still maintaining the important extracellular protein content.

Because detergents, such as Triton X-100, induce the activation of the pro-form of MMPs, ${ }^{26}$ the net proteolytic activity was compared in the two de-cellularization protocols using a gelatin substrate (DQ gelatin) (Fig. 2B). This substrate is cleaved by a broad range of proteases, including the gelatinases MMP-2 and MMP-9. Cleavage yields highly fluorescent peptides, whose fluorescence is proportional to the proteolytic activity. Two-way analysis of variance revealed significant detergent $(p=0.0006)$ and time $(p<0.0001)$ effects. The net gelatinase activity was significantly increased in the 24-h de-cellularization protocol, but not in the 50-h decellularization protocol, and was primarily derived from SDC-mediated activation. Increased MMP activation can result in degradation of important ECM proteins and therefore render the scaffold less favorable for cellular adherence and development. The proteolytic activity in control lungs rinsed with PBS for $50 \mathrm{~h}$ was comparable to de-cellularized lungs. Assay specificity is demonstrated by the reduced gelatinase activity in the presence of protease inhibitors. Taken together, these data indicated that the proteolytic activity is increased in the 24-h protocol, but results in the retention of ECM proteins comparable to or better than the more protracted protocol.

\section{Analysis of a 24-h de-cellularized scaffold by immunofluoresence}

Confocal microscopy of a de-cellularized lung that was subjected to the 24-h protocol compared with the normal lung revealed loss of CD31, which is expressed on endothelial cells and on peripheral leukocytes and platelets (Fig. 3A, B). The de-cellularized lung did not express MHC Class I, which is displayed on cell surfaces and is responsible for lymphocyte recognition and antigen presentation (Fig. 3C, D). In parallel with findings from the western blots and with what we have previously observed with the 50-h de-cellularization proto$\mathrm{Col}^{17}$ there was preservation of smooth muscle actin (Fig. 3E, F), collagen I (Fig. 3G, H), collagen IV (Fig. 3I, J), elastin (Fig. $3 \mathrm{~K}, \mathrm{~L}$ ), and laminin (Fig. 3M, N), which comprise the basement membrane and muscle layers surrounding vessels.

\section{Histology of 24-h de-cellularization with addition of coating agents}

After addition of either collagen I or Matrigel, gomori trichrome staining revealed an increase in collagen content compared to the de-cellularized scaffold alone (Fig. 4A, E, I, and $\mathrm{M}$ ). There was no obvious difference either in general architecture or in the loss of GAGs (Fig. 4B, F, J, and N; C, G, K, and $\mathrm{O}$ ). Addition of either coating agent qualitatively appeared to increase levels of elastin compared to de-cellularized. This may be due to nonspecific background staining of the added matrix (Fig. 4D, H, L, and P). 
A Normal Lung
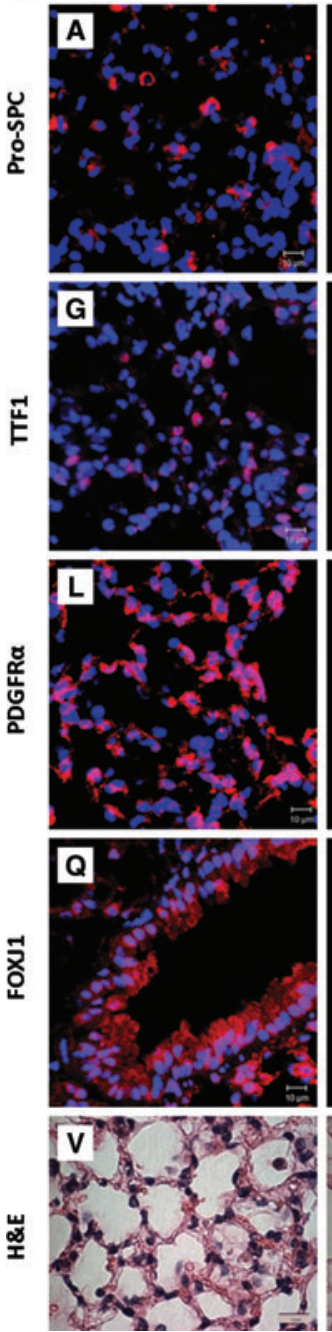
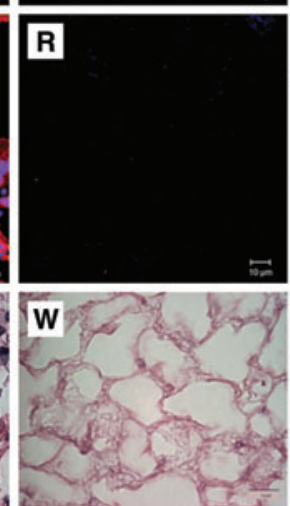

Seeded Lung
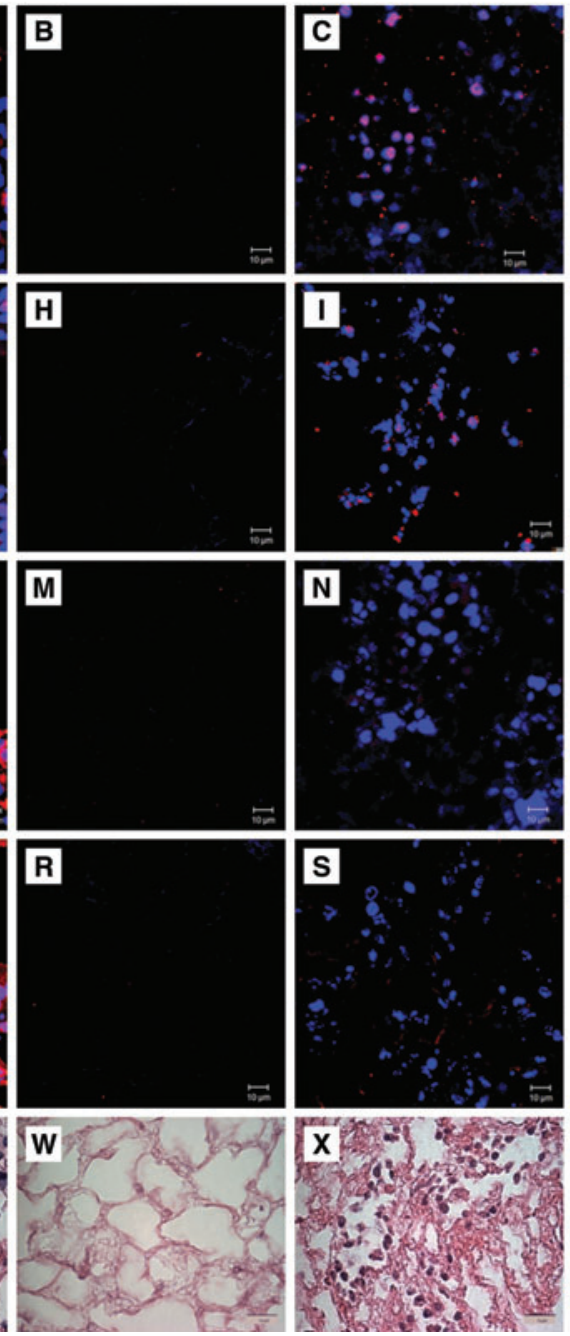

Seeded Collagen

Seeded Matrigel

$2^{\circ}$ Antibody Only
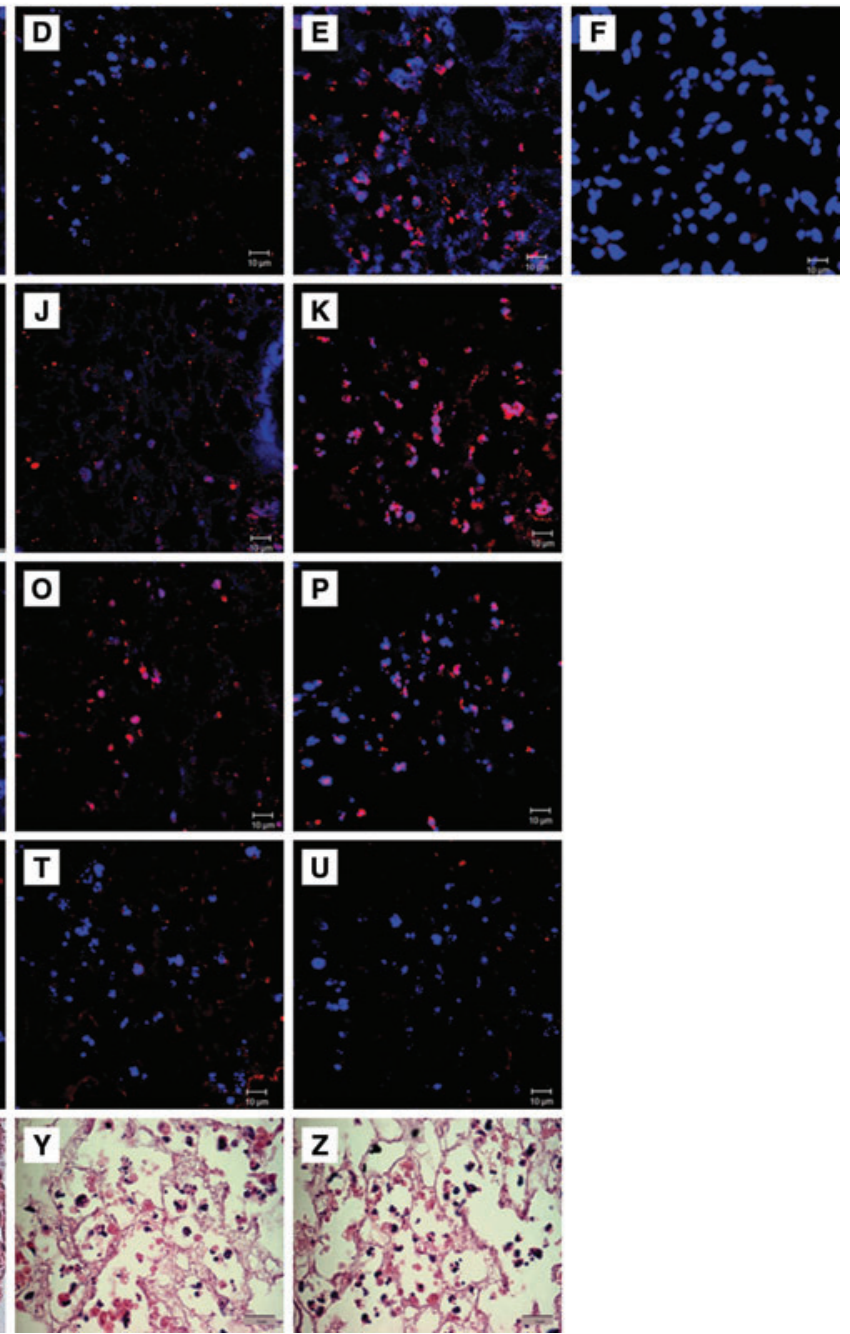

FIG. 6. Differentiated mESCs seeded into de-cellularized lungs in vitro show maintained expression, which increases and diversifies after implantation (A) Immunofluorescence and H\&E evaluation of a normal lung, decell lung, seeded, seeded and collagen-coated, and seeded and Matrigel-coated after incubation in vitro for $24 \mathrm{~h}$. Constructs were fixed and stained for Pro-SpC, TTF1, PDGFR $\alpha$, and FOXJ1 after incubation. (B) Immunofluorescence and H\&E evaluation of normal lung, decell lung, seeded- and implanted scaffold, collagen-coated/-seeded and collagen-implanted scaffold, and Matrigel-coated/seeded and Matrigel-implanted scaffold. Constructs were fixed and stained for Pro-SpC, TTF1, PDGFR $\alpha$, FOXJ1, and OCT4 after 14 days of subcutaneous implantation. Secondary antibody control was the same for all staining conditions. Representative images for all conditions are shown $(63 \times$ oil magnification [IF] and $100 \times$ oil magnification [H\&E]). (C) Graph demonstrates the positive cell count relative to the total cell count of the images found in (A). (D) Graph demonstrates the positive cell count relative to the total cell count of the images found in (B). Pro-SpC, pro-surfactant protein C; TTF1, thyroid transcription factor 1. Color images available online at www.liebertonline.com/tec

\section{Mechanical analysis of de-cellularized, seeded, and coated scaffolds}

Important characteristics of a lung scaffold would be the ability to withstand pressures generated by a ventilator. Additionally, properties like elasticity and resistance will be important. FlexiVent analyses demonstrated increased resistance and elastance and decreased compliance of rapidly de-cellularized lungs when compared with normal explanted lungs, similar to what we observed in the 50 -h protocol $^{17}$ (Fig. 5). These characteristics likely correspond, in part, to loss of surfactant and elastin. ${ }^{17}$ Seeding the scaffolds with differentiated mESCs or coating the de-cellularized lung with Matrigel did not ameliorate the increased stiffness ob- served in the de-cellularized lung scaffolds (Fig. 5). Upon coating the scaffolds with type I collagen, elastance and total lung resistance decreased, and compliance increased to levels that more closely mimic normal lung mechanics.

\section{Maintenance of differentiation of mESCs seeded into de-cellularized lungs in vitro}

Another important characteristic of de-cellarized scaffolds is the ability to support cellular differentiation. Murine ESCs that were induced to express TTF-1 and pro-SPC using our two-step differentiation protocol ${ }^{3}$ were inoculated by intratracheal administration and incubated for $24-48 \mathrm{~h}$ at $37^{\circ} \mathrm{C}$ in differentiation media with or without the addition of a 
B

B Normal Lung
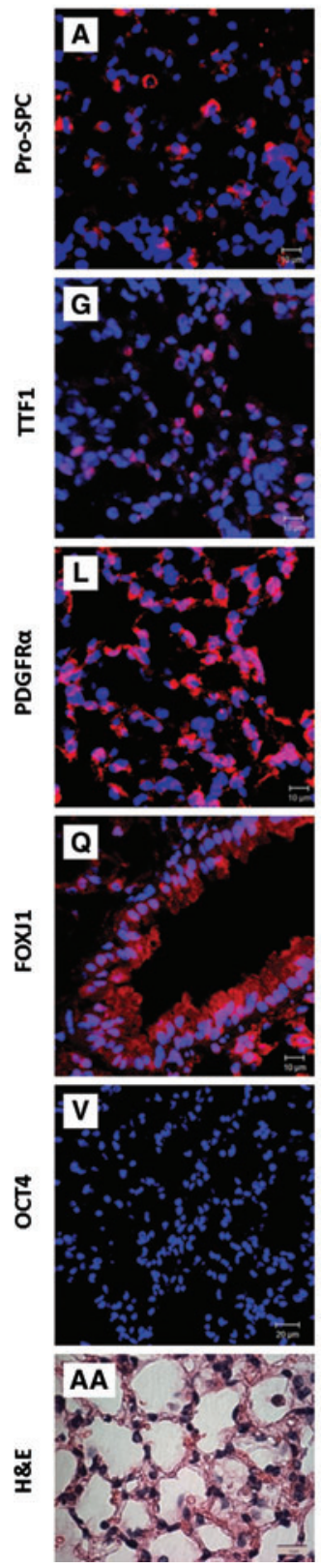
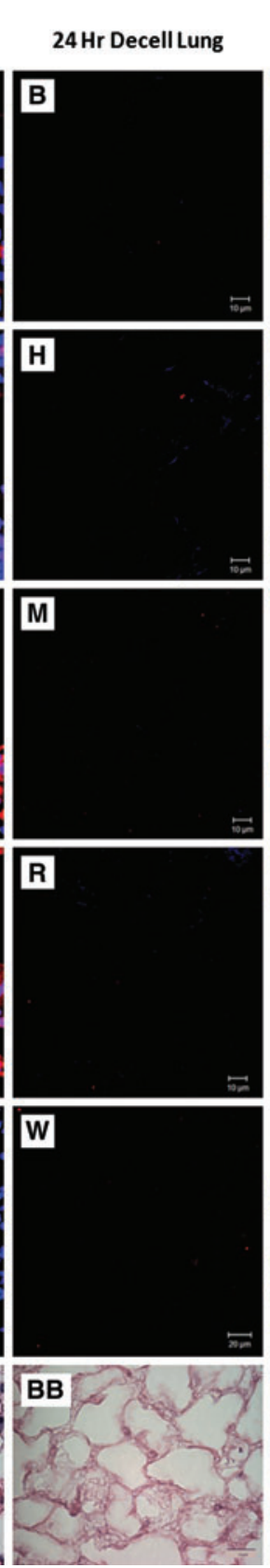

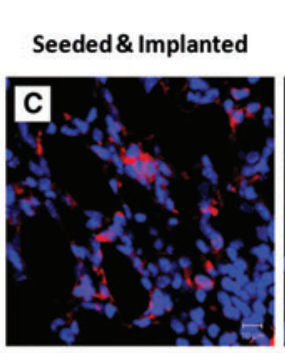

Seeded/Collagen \& Implanted
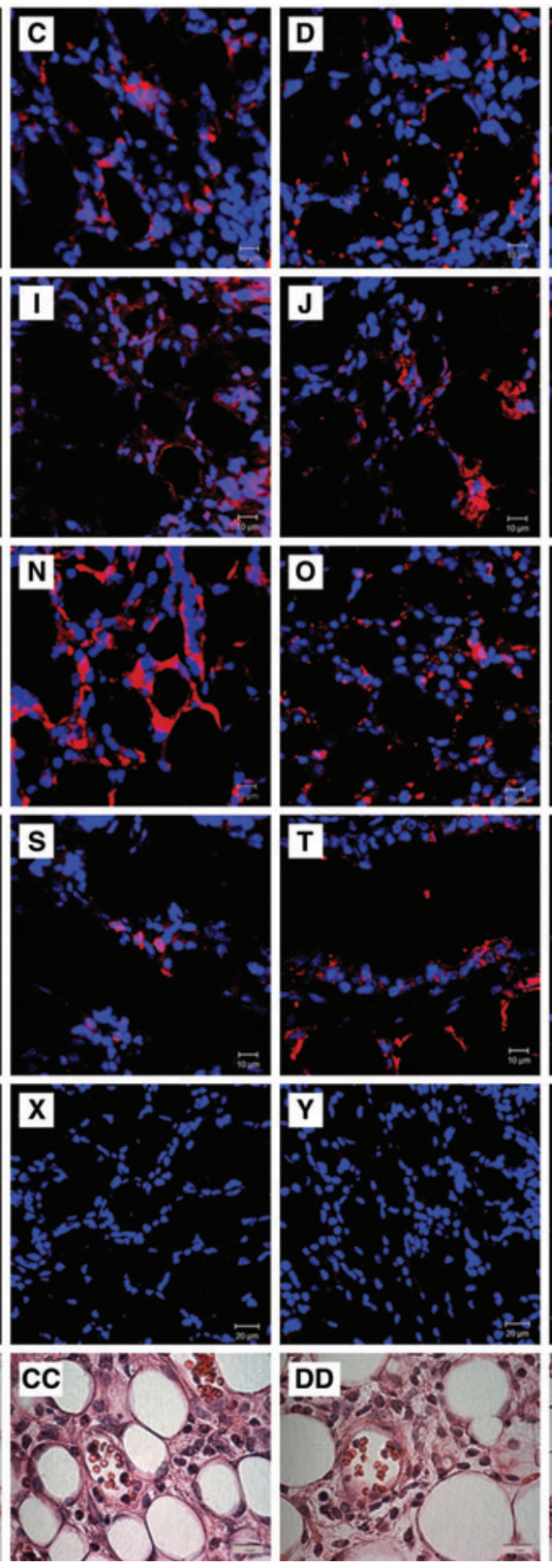

$2^{\circ}$ Antibody Only

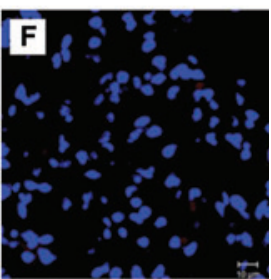

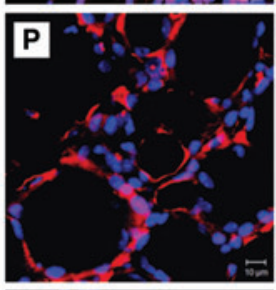
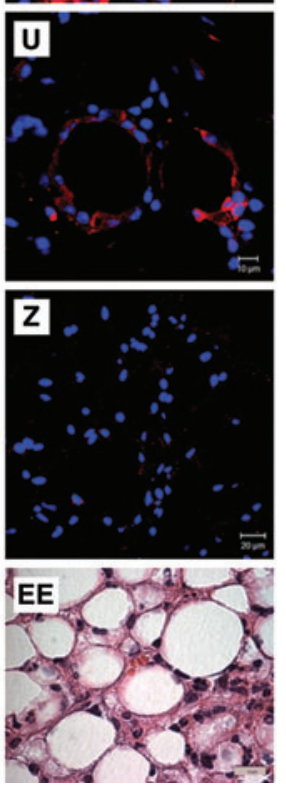

FIG. 6. (Continued).

coating agent before seeding. Pro-SPC, a marker of type II alveolar cell differentiation, appeared in the uncoated and Matrigel-coated conditions, but was absent in the collagen Icoated condition (Fig. 6A subpanels C, D, and E). The same trend was also observed in expression of the early lung developmental marker TTF1, where scaffolds uncoated or coated with Matrigel both demonstrated expression, and those coated in collagen I showed minimal expression (Fig. 6A subpanels I, J, and K). PDGFR $\alpha$ plays a major role in lung development and is also expressed during development in the mesenchyme. ${ }^{10}$ Some expression of PDGFR $\alpha$ was observed in the Matrigel- and collagen I-coated scaffolds (Fig. 6A subpanels $\mathrm{N}, \mathrm{O}$, and $\mathrm{P}$ ), but not in the seeded-only scaffold. FOXJ1 expression, a ciliated proximal epithelial cell marker, was absent in all conditions (Fig. 6A subpanels $\mathrm{S}, \mathrm{T}$, and $\mathrm{U}$ ). As expected, the de-cellularized lung was absent for all markers of cellular differentiation, as there were no cells (Fig. 6A subpanels B, H, M, and R). The numbers of positive cells in these images were quantified to analyze the expression for each marker in the various coating conditions. (Fig. 6C).

Maintenance of differentiation of mESCs seeded into de-cellularized lungs and implanted in vivo

Another important feature of a viable lung scaffold will be its ability to integrate within a host while maintaining cellular differentiation following implantation. De-cellularized 


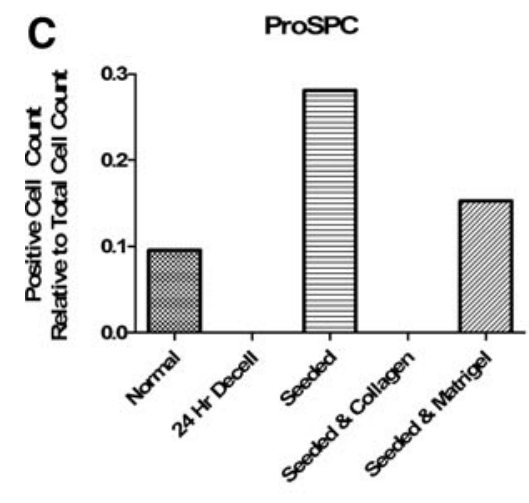

PDGFR

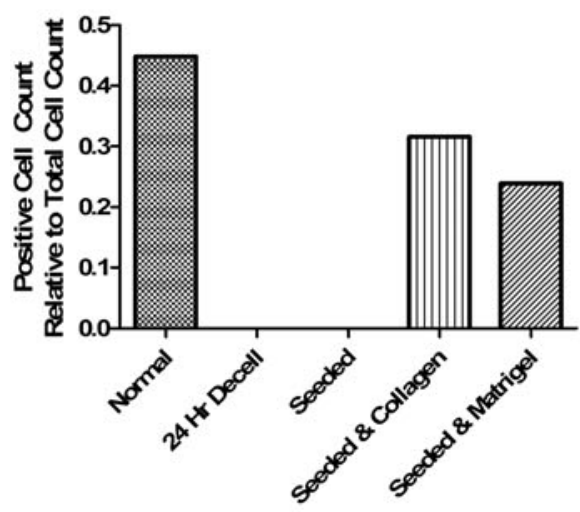

D ProspC

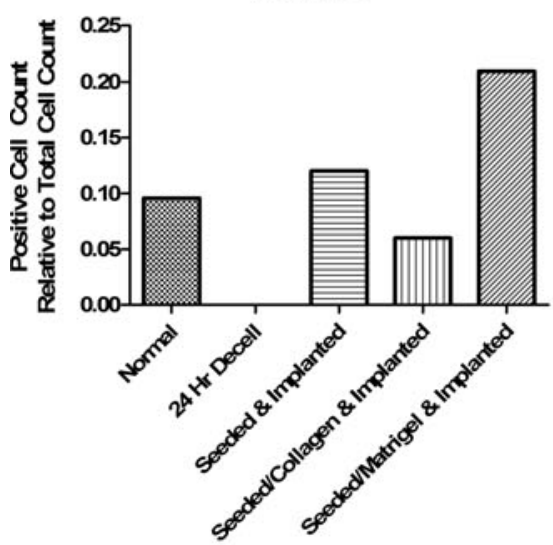

PDGFR

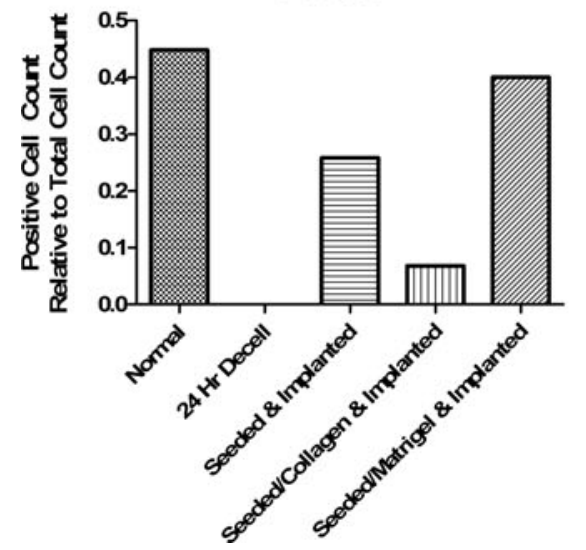

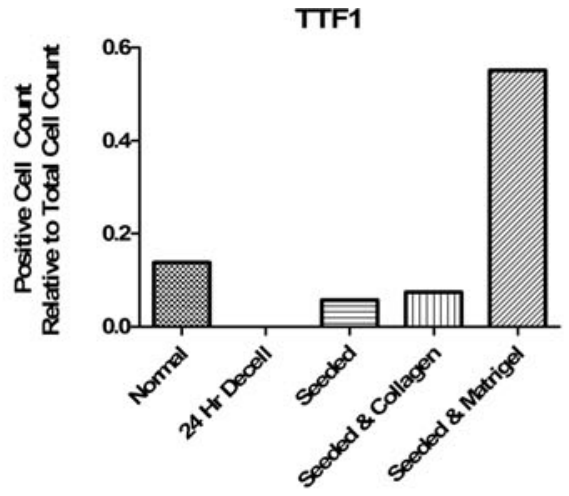

FOXN1

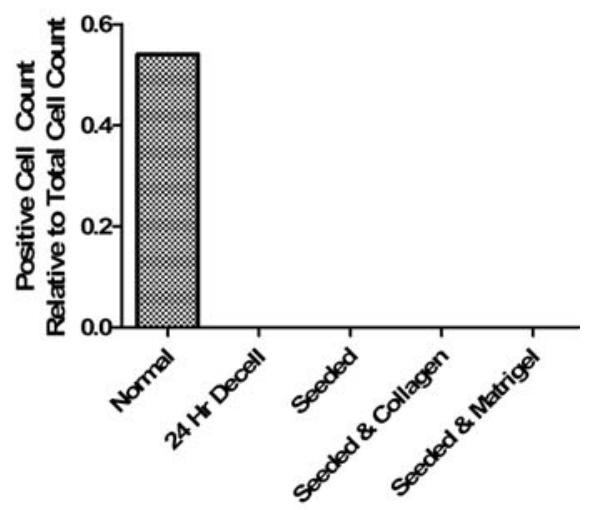

TTF1

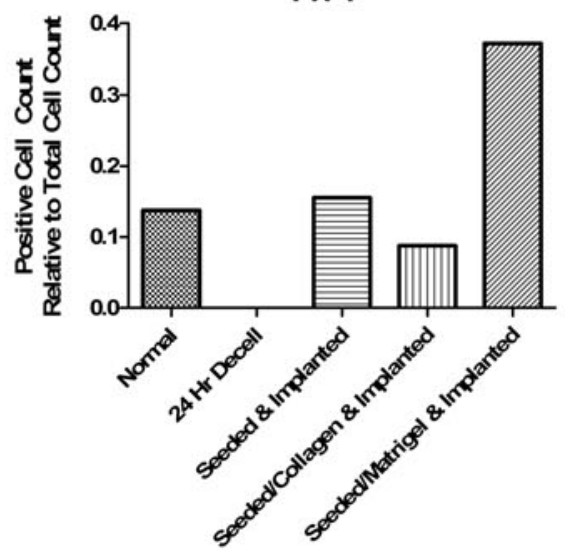

FOXJ1

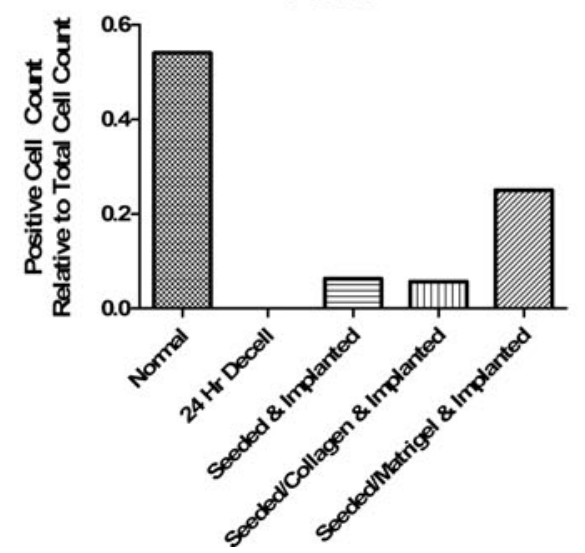

FIG. 6. (Continued). 
lungs were seeded with differentiated mESCs with or without the use of a coating agent. The scaffolds were subsequently implanted subcutaneously for 14 days in vivo and were evaluated via immunofluorescence and H\&E staining. Immunofluorescence of Pro-SPC (Supplementary Fig. S1, Fig. 6B, subpanels C-E), TTF1 (Fig. 6B, subpanels I-K), PDGFR $\alpha$ (Fig. 6B, subpanels N-P), and FOXJ1 (Fig. 6B, subpanels S-U) was qualitatively brighter in scaffolds seeded and implanted and seeded/Matrigel and implanted compared to seeded/ collagen and implanted. Expression of FOXJ1 after implantation suggests development of proximal airway cells within the scaffold (Fig. 6B subpanels S-U). A marker of pluripotency, Oct 3/4, was conspicuously absent in all conditions after implantation. This is not surprising given previously published results of loss of OCT 3/4 expression when cells were treated with FGF2. ${ }^{3}$ The de-cellularized lung was absent for all cellular markers as expected (Fig. 6B subpanels B, H, M, and $\mathrm{R})$. The positive cell number for each marker was quantified and graphed to further demonstrate which coating conditions were favorable for airway cell maintenance (Fig. 6D).

\section{Neovascularization of implanted scaffolds}

Finally, another important characteristic of engineering lung scaffolds is the ability to integrate with the host and induce neovascularization to promote long-term survival of the graft. Blood vessels stained positive for both Isolectin B4 (red) (Jackson Immunresearch) and FITC dextran (green) in the seeded and seeded/coated scaffold conditions (Fig. 7). Red blood cells were also present in these H\&E sections (arrows), demonstrating neovascularization of the constructs after 14 days of implantation.

\section{Discussion}

The ability to utilize an acellular biomimetic scaffold to support differentiated stem cells would be invaluable in potentially developing a treatment for pulmonary diseases. The rapid creation of a relevant scaffold will increase the efficiency of the clinical delivery and availability. Herein, we describe a $24-h$ process for de-cellularization of murine lungs and compare the results of this fast de-cellularization process to the original $50-\mathrm{h}$ de-cellularization procedure. Importantly, a scaffold should have the ability to support appropriate growth and differentiation of inoculated cells. We demonstrate the ability to seed this scaffold with differentiated mESCs and implant them subcutaneously with maintenance of differentiation and neovascularization.

Several recent reports have demonstrated methods of de-cellularizing the native lung, ${ }^{10,14,17-20,27}$ requiring between $50 \mathrm{~h}$ and 6 weeks. Each of these utilized different detergent-, chemical-, and physical-based de-cellularization approaches. However, the optimal means of de-cellularizing lungs while maintaining architecture is not yet clear. Further, it is unknown what optimization of lung de-cellularization entails. Presumably, this includes maintenance of gross and microscopic anatomy as well as preservation of key ECM proteins. In each of the published recent studies, varying amounts of ECM proteins are preserved. Evaluation of our 24-h de-cellularization process via histology, immunofluorescence, and western blot analysis demonstrates lung scaffolds with preservation of key ECM proteins collagen I, collagen IV, fibronectin, and laminin, but with loss of nuclear proteins. Scanning and trans- mission electron microscopy confirmed the removal of cells from the scaffold with minimal basement membrane disruption, which is similar to other reports. ${ }^{17}$ Each of these ECM proteins plays roles in embryonic development, cell proliferation and differentiation, as well as cell adhesion and migration. ${ }^{11,13,14,27,28}$ The results we obtained after de-cellularization are similar to those of others who report preservation of most ECM proteins, except elastin and GAGs. ${ }^{14,17-19}$ Elastin is a critical component of the lung interstitium, providing the property of recoil to the vascular, conducting airway, and terminal airspace compartments of the lung. ${ }^{12}$ Future protocols need to emphasize the preservation of elastin in these scaffolds. Although GAGs contribute to the viscoelasticity of the lungs, they can also can be immunostimulatory and, as suggested by Price et al., their loss may contribute to an immuno-privileged status, which may be beneficial. ${ }^{14}$

Previously published reports have indicated that evaluation of de-cellularized scaffolds for activation of MMPs is essential. Although MMPs are largely thought of as ECM proteases, it is also well accepted that they can degrade intracellular substrates as well. ${ }^{29,30}$ Moreover, cellular lysis in lung de-cellularization protocols will expose the extracellular environment to cytosolic proteases (e.g., serine, aspartic, and cysteine) that they would normally never encounter. This may adversely affect cell adhesion, differentiation, and survival of inoculated stem cells. We observed a profound increase in the gelatinase activity following the $24-\mathrm{h}$ protocol as compared to Triton, SDC, PBS, or the full 50-h protocol. ${ }^{17}$ Subsequently treating with inhibitors did result in a decrease in the gelatinase activity following the 24-h de-cellularization protocol; however, the decrease was more profound in the other de-cellularization approaches that were investigated. The relevance of these data is currently unclear, but may, in fact, affect long-term cell adherence, viability, and development when utilizing these scaffolds. ${ }^{17}$

Theoretically, the mechanical properties of the scaffold, such as elastance and resistance, will be clinically relevant. We and others noted that de-cellularized lungs have decreased compliance and increased resistance and elastance. This is likely due to the loss of the surfactant, which normally decreases alveolar surface tension, as well as the loss of cells and matrix components. ${ }^{14,17}$ Coating the de-cellularized lungs with Matrigel did not reverse changes in lung mechanics, but coating with collagen I resulted in a decrease in total lung resistance and elastance, as well as an increase in compliance. We would have expected the addition of collagen to increase resistance and decrease compliance, similar to what is seen in pulmonary fibrosis. Further investigation is needed to characterize the use of coating agents and addition of exogenous proteins such as a surfactant on lung mechanics in de-cellularized scaffolds. Another variable that may affect mechanics includes the cell-seeding density. In our hands, seeding the scaffold with a relatively low number of differentiated murine ESCs did not improve lung compliance as reported by others. ${ }^{14}$ This likely reflects too few cells to influence lung mechanics.

Another important characteristic of a lung scaffold is its ability to support cellular growth and differentiation. Cortiella et al. described the use of the de-cellularized lung scaffolds to induce differentiation of undifferentiated ESCs into those with immunophenotypes of a variety of lungspecific cells. ${ }^{10}$ In our studies, we predifferentiated our cells 
FIG. 7. Neovascularization develops in scaffolds that were subcutaneously implanted for 14 days with some evidence of perfusion. Evaluation of vascular development within the scaffolds after 14 days of subcutaneous implantation. FITC dextran was injected before harvest, and scaffolds were stained with Isolectin B4 to visualize the vessels and assess for perfusion from the host. Representative images for all conditions are shown $(63 \times$ oil magnification [IF] and $100 \times$ oil magnification [H\&E]). Arrows indicating blood vessels within the implant. FITC, fluorescein iso thiocyanate; DAPI, 4'6diamidino-2-phenylindole. Color images available online at www.liebertonline.com/ tec
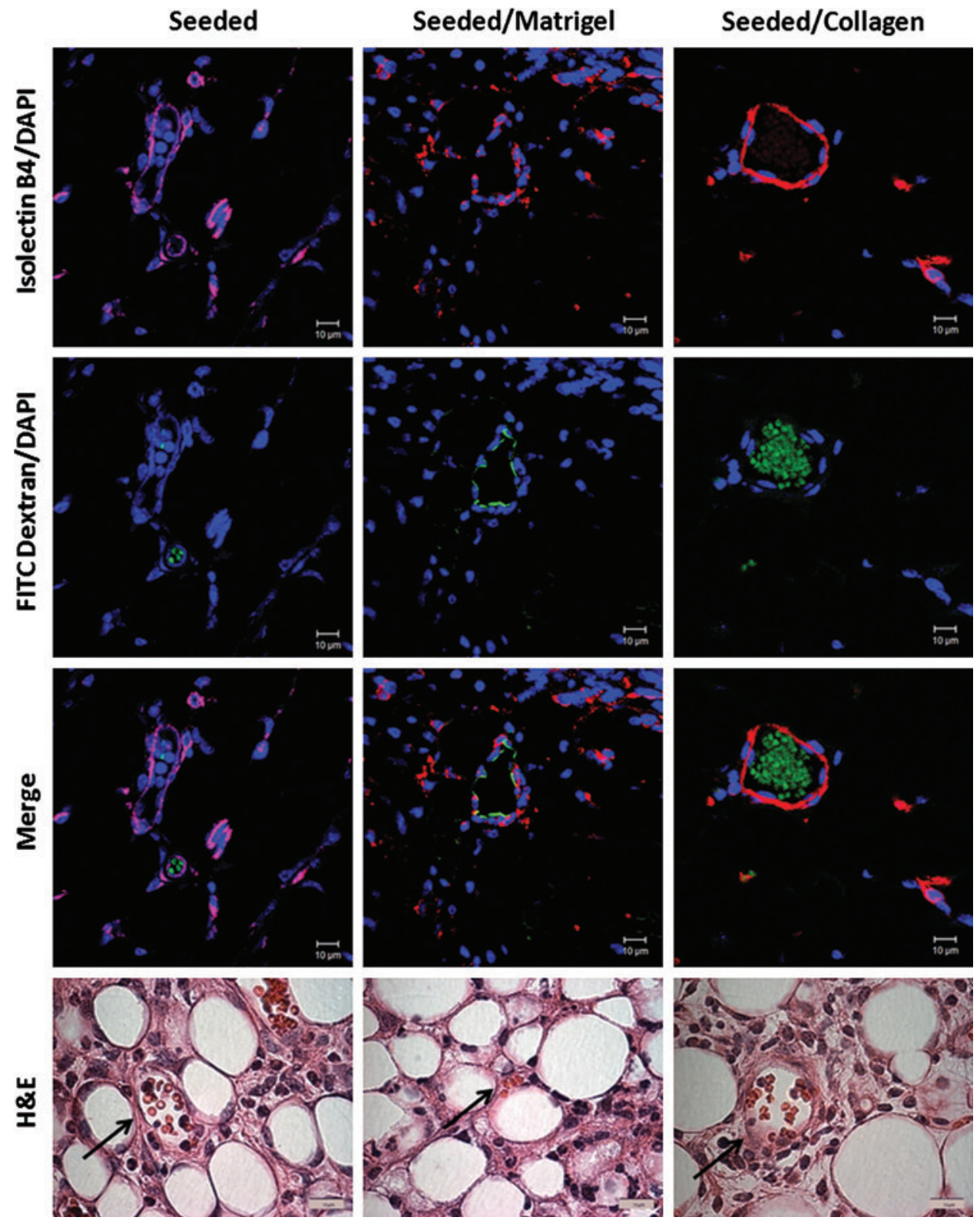

into distal airway cells with phenotypic characteristics of type II alveolar epithelial cells and seeded them into the decellularized lung scaffolds. The purpose was to demonstrate that the de-cellularized scaffolds could maintain differentiation of these cells, including continued pro-SpC and TTF1 expression. We did identify improved expression of pro-SpC, TTF1, PDGFR $\alpha$, and FOXJ1 in our implanted, seeded scaffolds compared to seeded scaffolds in culture. Interestingly, FOXJ1 expression was absent in culture, but was present after implantation. This underscores the heterogeneity and plasticity of our differentiated stem cells and the importance of an in vivo environment. We are the first to implant predifferentiated mESCs within a de-cellularized construct and show maintenance of lung-specific differentiation. Further, blood vessel formation with suggestion of blood flow from the host was noted after 14 days of implantation. This will be critical in the viability of the construct and as a model system to follow in vivo behaviors of cells inoculated into the de- cellularized scaffolds. Neovascularization of the scaffolds coated with collagen or Matrigel stained positive for both Isolectin B4 and FITC Dextran. The seeded-only condition did show neovascularization as demonstrated by Isolectin B4 staining; however, the amount of FITC dextran staining was diminished compared to the coated conditions. This may represent less vascular in-growth from the host compared to the coated conditions and could reflect exogenous growth factors present in the Matrigel.

Although we did not observe any obvious teratoma formation, our experiments were relatively short term. Longer implantation of re-cellularized scaffolds should be the focus of future research studies.

\section{Conclusion}

Use of a rapid detergent-based de-cellularization protocol results in a scaffold that maintains architecture and critical 
ECM components and enables maintenance of mESC differentiation both in vitro and after subcutaneous implantation. Neovascularization of implanted constructs will facilitate a long-term study to follow the potential development of a functional lung tissue. These studies will provide a strong basis for the potential clinical use of ex vivo-engineered lung tissues derived using de-cellularized whole lungs.

\section{Acknowledgments}

The authors would like to acknowledge the animal care staff at the University of Connecticut Health Center as well as Dr. Adam Matson for his contributions in lung mechanics. We also would like to thank Camilo A. Moncada, Ph.D., for his assistance in critically reviewing this article. Lung-mechanic studies were supported by NIH 3K08AI071918-02S1. The authors would also like to thank Peter Lelkes, Ph.D., for critical review of this article. The authors would also like to acknowledge the staff of the Offices of Animal Care Management at the University of Vermont and Katie Polakowski and Kevin Weiss for valuable contributions to the experimental studies. Studies were supported by NIH ARRA RC4HL106625 (D.J.W.) and NHLBI R21HL094611 (D.J.W.). Facilities and equipment were supported by the UVM Lung Biology COBRE (NIH NCRR P20 RR-155557), NIH Neuroscience COBRE (NIH NCRR P20 RR016435), and the Vermont Cancer Center DNA Analysis facility (NIH P30 CA22435).

Support sources:

NIH Award: 3K08AI071918-02S1- SCIREQ FlexiVent Grant (A.M.)

NIH Award: 1RO1HL104258-01 (C.M.F)

NIH Award: ARRA RC4HL106625 (D.J.W. and C.M.F)

NHLBI Award: R21HL094611 (D.J.W.)

\section{Disclosure Statement}

No competing financial interests exist for the authors of this article.

\section{References}

1. Foundation MoD. Preterm Birth Overview. Quick Facts. White Plains: March of Dimes Foundation, 2010.

2. Leinwand, M.J., Tefft, J.D., Zhao, J., Coleman, C., Anderson, K.D., and Warburton, D. Nitrofen inhibition of pulmonary growth and development occurs in the early embryonic mouse. J Pediatr Surg 37, 1263, 2002.

3. Roszell, B., Mondrinos, M.J., Seaton, A., Simons, D.M., Koutzaki, S.H., Fong, G.H., et al. Efficient derivation of alveolar type II cells from embryonic stem cells for in vivo application. Tissue Eng Part A 15, 3351, 2009.

4. Samadikuchaksaraei, A., and Bishop, A.E. Derivation and characterization of alveolar epithelial cells from murine embryonic stem cells in vitro. Methods Mol Biol 330, 233, 2006.

5. Samadikuchaksaraei, A., and Bishop, A.E. Effects of growth factors on the differentiation of murine ESC into type II pneumocytes. Cloning Stem Cells 9, 407, 2007.

6. Wang, D., Haviland, D.L., Burns, A.R., Zsigmond, E., and Wetsel, R.A. A pure population of lung alveolar epithelial type II cells derived from human embryonic stem cells. Proc Natl Acad Sci U S A 104, 4449, 2007.
7. Wang, D., Morales, J.E., Calame, D.G., Alcorn, J.L., and Wetsel, R.A. Transplantation of human embryonic stem cellderived alveolar epithelial type II cells abrogates acute lung injury in mice. Mol Ther 18, 625, 2010.

8. Rippon, H.J., Polak, J.M., Qin, M., and Bishop, A.E. Derivation of distal lung epithelial progenitors from murine embryonic stem cells using a novel three-step differentiation protocol. Stem Cells 24, 1389, 2006.

9. Lin, Y.M., Zhang, A., Rippon, H.J., Bismarck, A., and Bishop, A.E. Tissue engineering of lung: the effect of extracellular matrix on the differentiation of embryonic stem cells to pneumocytes. Tissue Eng Part A 16, 1515, 2010.

10. Cortiella, J., Niles, J., Cantu, A., Brettler, A., Pham, A., Vargas, G., et al. Influence of acellular natural lung matrix on murine embryonic stem cell differentiation and tissue formation. Tissue Eng Part A 16, 2565, 2010.

11. Fernandes, H., Mentink, A., Bank, R., Stoop, R., van Blitterswijk, C., and de Boer, J. Endogenous collagen influences differentiation of human multipotent mesenchymal stromal cells. Tissue Eng Part A 16, 1693, 2010.

12. Mariani, T.J., Sandefur, S., and Pierce, R.A. Elastin in lung development. Exp Lung Res 23, 131, 1997.

13. Nguyen, N.M., and Senior, R.M. Laminin isoforms and lung development: all isoforms are not equal. Dev Biol 294, 271, 2006.

14. Price, A.P., England, K.A., Matson, A.M., Blazar, B.R., and Panoskaltsis-Mortari, A. Development of a decellularized lung bioreactor system for bioengineering the lung: the matrix reloaded. Tissue Eng Part A 16, 2581, 2010.

15. Cortiella, J., Nichols, J.E., Kojima, K., Bonassar, L.J., Dargon, P., Roy, A.K., et al. Tissue-engineered lung: an in vivo and in vitro comparison of polyglycolic acid and pluronic F-127 hydrogel/somatic lung progenitor cell constructs to support tissue growth. Tissue Eng 12, 1213, 2006.

16. Lwebuga-Mukasa, J.S., Ingbar, D.H., and Madri, J.A. Repopulation of a human alveolar matrix by adult rat type II pneumocytes in vitro. A novel system for type II pneumocyte culture. Exp Cell Res 162, 423, 1986.

17. Daly, A.B., Wallis, J.M., Borg, Z.D., Bonvillain, R.W., Deng, B., Baliff, B.A., et al. Initial binding and re-cellularization of decellularized mouse lung scaffolds with bone marrow-derived mesenchymal stromal cells. Tissue Eng Part A 18, 1, 2012.

18. Ott, H.C., Clippinger, B., Conrad, C., Schuetz, C., Pomerantseva, I., Ikonomou, L., et al. Regeneration and orthotopic transplantation of a bioartificial lung. Nat Med 16, 927, 2010.

19. Petersen, T.H., Calle, E.A., Zhao, L., Lee, E.J., Gui, L., Raredon, M.B., et al. Tissue-engineered lungs for in vivo implantation. Science 329, 538, 2010.

20. Song, J.J., Kim, S.S., Liu, Z., Madsen, J.C., Mathisen, D.J., Vacanti, J.P., et al. Enhanced in vivo function of bioartificial lungs in rats. Ann Thorac Surg 92, 998, 2011.

21. Roszell, B., Seaton, A., Fong, G.H., and Finck, C.M. Cellbased therapy improves cell viability and increases airway size in an explant model. Exp Lung Res 35, 501, 2009.

22. Lluri, G., Langlois, G.D., Soloway, P.D., and Jaworski, D.M. Tissue inhibitor of metalloproteinase-2 (TIMP-2) regulates myogenesis and beta1 integrin expression in vitro. Exp Cell Res 314, 11, 2008.

23. Lluri, G., Langlois, G.D., McClellan, B., Soloway, P.D., and Jaworski, D.M. Tissue inhibitor of metalloproteinase-2 (TIMP2) regulates neuromuscular junction development via a beta1 integrin-mediated mechanism. J Neurobiol 66, 1365, 2006.

24. Bates, J.H., Cojocaru, A., Haverkamp, H.C., Rinaldi, L.M., and Irvin, C.G. The synergistic interactions of allergic lung 
inflammation and intratracheal cationic protein. Am J Respir Crit Care Med 177, 261, 2008.

25. Al Jamal, R., Roughley, P.J., and Ludwig, M.S. Effect of glycosaminoglycan degradation on lung tissue viscoelasticity. Am J Physiol Lung Cell Mol Physiol 280, L306, 2001.

26. Nagase, H. Activation mechanisms of matrix metalloproteinases. Biol Chem 378, 151, 1997.

27. Shamis, Y., Hasson, E., Soroker, A., Bassat, E., Shimoni, Y., Ziv, T., et al. Organ-specific scaffolds for in vitro expansion, differentiation, and organization of primary lung cells. Tissue Eng Part C Methods 17, 861, 2011.

28. Andrades, J.A., Nimni, M.E., Han, B., Ertl, D.C., Hall, F.L., and Becerra, J. Type I collagen combined with a recombinant TGF-beta serves as a scaffold for mesenchymal stem cells. Int J Dev Biol Suppl 1, 107S, 1996.

29. McCawley, L.J., and Matrisian, L.M. Matrix metalloproteinases: they're not just for matrix anymore! Curr Opin Cell Biol 13, 534, 2001.
30. Cauwe, B., and Opdenakker, G. Intracellular substrate cleavage: a novel dimension in the biochemistry, biology and pathology of matrix metalloproteinases. Crit Rev Biochem Mol Biol 45, 351, 2010.

Address correspondence to: Christine Finck, M.D. Department of Surgery Connecticut Children's Medical Center 282 Washington St. Hartford, CT 06106

E-mail: cfinck@ccmckids.org

Received: October 20, 2011

Accepted: February 15, 2012

Online Publication Date: April 12, 2012 